\title{
Analysis of Multiple-Input-Multiple-Output (MIMO) System with Transmit and Receive Diversity
}

\author{
S.Taruna \\ Computer Science Department \\ Banasthali University \\ Jaipur, India
}

\author{
Ikpreet Kaur \\ Information Technology Department \\ Banasthali University \\ Jaipur, India
}

\begin{abstract}
The gist of Multiple-Input-Multiple-Output (MIMO) technique is its ability to provide increased channel rate with the use of multiple antennas, thus increasing spatial diversity gain. Correspondingly, there is a noticeable amount of increase in the number of users in wireless systems equipped with MIMO technology. In this work, the performance of MIMO Systems have been analyzed with transmit and receive diversity. The performance of $2 \times 2,4 \times 4,8 \times 8$ and $12 \times 12$ MIMO Systems have been compared by performing simulations in MATLAB on the basis of channel rate.
\end{abstract}

\section{General Terms}

SNR is the signal-to-noise ratio of the channel; MIMO stands for Multiple-Input-Multiple-Output.

\section{Keywords}

MIMO, SISO, Channel Capacity, Wireless System

\section{INTRODUCTION}

Wireless System has an endless quest for higher capacity and improved quality as it provides people and machines to communicate with each other irrespective of their location. Fourth generation wireless communication has a very famous slogan "always best connected" which means that the wireless devices should connect to the network at that moment that is best for the user. MIMO is a novel approach for the next generation wireless systems as it provides distinctive solutions for high performance network.

Multiple-Input-Multiple-Output also known as MIMO is a very powerful technique for wireless system. MIMO structure consist of multiple antennas at both transmitter and receiver end for improving the communication performance. It provides higher capacity, throughput with improved quality of service without increasing the transmitted power of antennas [1].

The benefits provided by MIMO in wireless network are array gain, multiplexing gain and spatial diversity [2]. Array gain is the gain in signal-to-noise ratio due to the use of multiple antennas which leads to increased range and coverage [3].

MIMO offers multiplexing gain by transmitting multiple independent data streams which leads to linear increase in throughput.

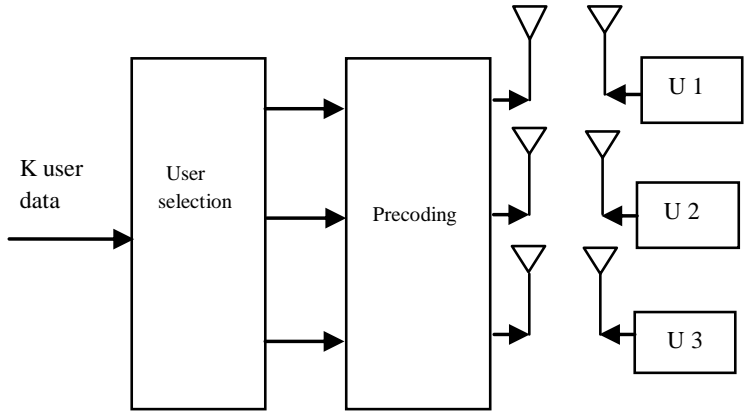

Fig 1: Multiplexing gain in MIMO

Spatial Diversity improves the robustness and capacity of the wireless system by forming multiple paths between sender and receiver [4].

The contribution of this article is to provide better understanding of the MIMO System and its channel capacity. This article is organized as follows. In section 1, it explains the general framework of the MIMO Systems. Section 2 gives the overview of the propagation model of the MIMO. Section 3 , discusses the channel capacity of the MIMO Systems.

\section{PROPAGATION MODEL OF MIMO}

MIMO propagation model consist of transmitted data, modulator, transmitting and receiving antennas, demodulator and wireless channel as shown in figure 2 . The data that is to be transmitted is divided into multiple streams and is then coded on different transmit antennas.

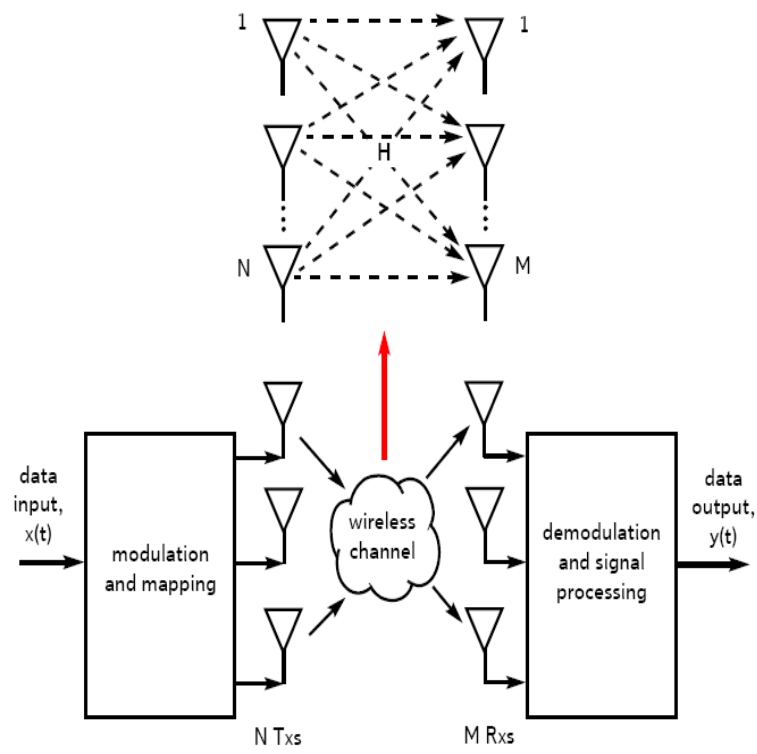

Fig: 2 Propagation Model of MIMO System 
In ideal MIMO System, the $\mathrm{N}$ kbps of bandwidth allocated to the complete MIMO system is divided between all the users equally and each user get $1 / \mathrm{N}$ of the total bandwidth.

To identify the independent signals that are transmitted in MIMO Systems, a channel matrix $\mathrm{H}$ is formed as shown in equation (1). At the destination end the receiver identify the channel matrix and individual transmitted signals are separated and estimated.

$$
H=\left[\begin{array}{ll}
a 11 & a 12 \\
a 21 & a 22
\end{array}\right]
$$

In general, the size of channel matrix is equal to Txs * Rxs where Txs is the number of transmitting antenna and Rxs is the number of receiving antenna[5] and MIMO System can be represented as (Txs,Rxs) elements. Here aij represents the single-input-single-output channel between $\mathrm{i}^{\text {th }}$ receive and $\mathrm{j}^{\text {th }}$ transmit antenna pair.

Mathematically, MIMO System can be represented as:

$$
y=H x+n
$$

where $y$ is the received signal vector, $H$ is the channel matrix of size Txs*Rxs, $x$ is the Rxs*1 transmit signal vector and $n$ is the Txs*1 noise additive white Gaussian noise vector.

Generally, the rank of MIMO channel can be defined by the algebraic rank of the Txs*Rxs channel matrix. Evidently, the rank of channel matrix is equal to or less than the number of transmitting antenna and number of receiving antenna. Thus, the number of independent signals that can be transmitted through a MIMO System is at most equal to the rank of channel matrix.

\section{CAPACITY OF MIMO CHANNELS}

An abstract communication system is considered to introduce the concept of capacity, where sequences of bits are transmitted to the receiver. In the case of single-input-singleoutput (SISO) channel, encoder at the transmitter side convert the bits to be transmitted into the codeword such as b bits are mapped into codeword $\{\mathrm{x}(1), \mathrm{x}(2), \ldots \ldots \ldots . . . \mathrm{x}(\mathrm{n})\}$. These codeword are then send to the receiver. At the receiver side, decoder translates the received codeword to recover the original message. [6]
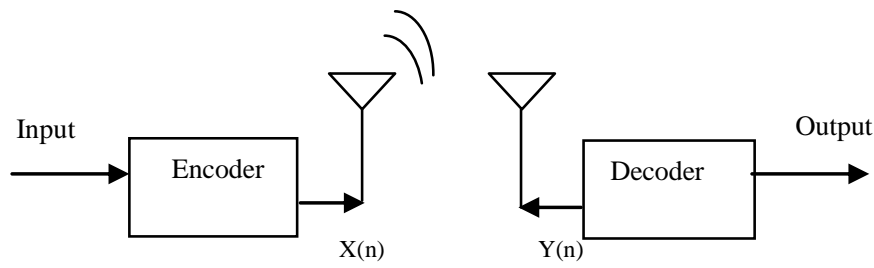

Fig3: Communication System of SISO

In figure 3 input bits represent the data to be transmitted, output bits stands for the original message for the receiver and $\mathrm{X}$ (n) symbolizes the codeword.

In Multiple-Input-Multiple-Output (MIMO) Systems the space dimension is added because of the use of multiple antennas at the both ends of the communication system. Encoder maps the data across time as in SISO System and as well as across space.

$$
\left[\begin{array}{ccc}
x 11 & \ldots & x 1 N \\
\vdots & \ddots & \vdots \\
x 1 M & \cdots & x M N
\end{array}\right]
$$

The two dimensional matrix in equation (3) represents the codeword data which is produced by the encoder at the transmitter side. Here $x M N$ represents data transmitted by $\mathrm{M}^{\text {th }}$ antenna and received by $\mathrm{N}^{\text {th }}$ antenna.

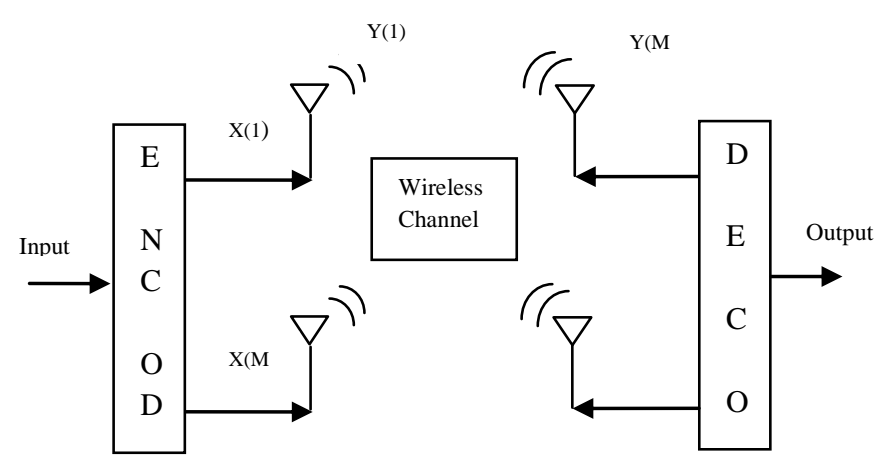

Fig 4: Communication of MIMO System

In figure $4, \mathrm{X}(1)$ and $\mathrm{X}(\mathrm{M})$ represents the transmitted codeword where $X(1)=\{x(11), x(12), \ldots \ldots x(1 N)\}$ that is the codeword transmitted by first antenna and $X(M)=\{x(1 M)$, $\mathrm{x}(2 \mathrm{M}), \ldots \ldots . . \mathrm{x}(\mathrm{MN})$ that is the codeword transmitted by $\mathrm{M}^{\text {th }}$ antenna.

\subsection{MIMO System Capacity when Channel is Constant}

Channel capacity of MIMO is the measure of the maximum amount of information that can be transmitted over a channel and received with a negligible probability of error at the receiver.[7]The capacity of the MIMO System when transmitter and receiver are aware of the channel matrix is denoted as:

$$
C=\max \log \left|I^{N}+H Q H^{\rho}\right|
$$

where $\mathrm{H}$ represents the channel matrix, $\mathrm{H}^{\mathrm{p}}$ represents the transpose of the channel matrix and $\mathrm{Q}$ is the covariance matrix.

\section{RESULTS AND SIMULATION}

In this paper, it compared the MIMO Systems for a range of antenna configurations by plotting a probability versus channel rate graph. This section is divided into five cases. In each case the graph has been plotted for different values of SNR such as $0 \mathrm{db}, 10 \mathrm{db}, 20 \mathrm{bd}, 40 \mathrm{db}$ and $60 \mathrm{db}$ and for each value of SNR it compared the performance by computing the channel rate for $2 \times 2,4 \times 4,8 \times 8$ and $12 \times 12$ antenna configurations in MIMO Systems. 
4.1 Case 1: Analysis of MIMO Systems for $\mathrm{SNR}=0 \mathrm{db}$

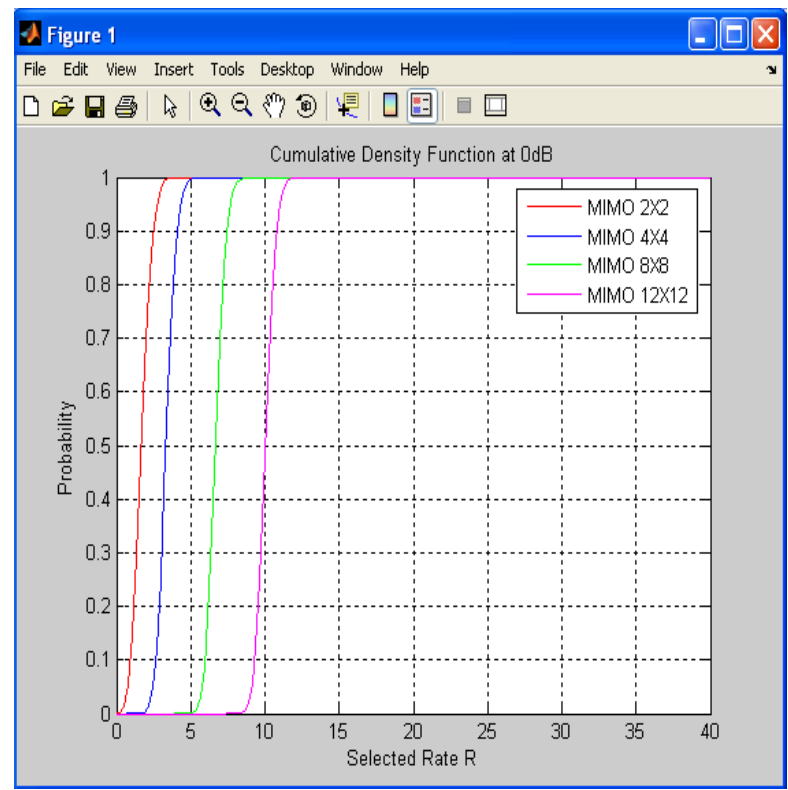

Fig 5: Comparison of MIMO Systems when SNR = 0 db

\subsection{Case 2: Analysis of MIMO Systems for $\mathrm{SNR}=10 \mathrm{db}$}

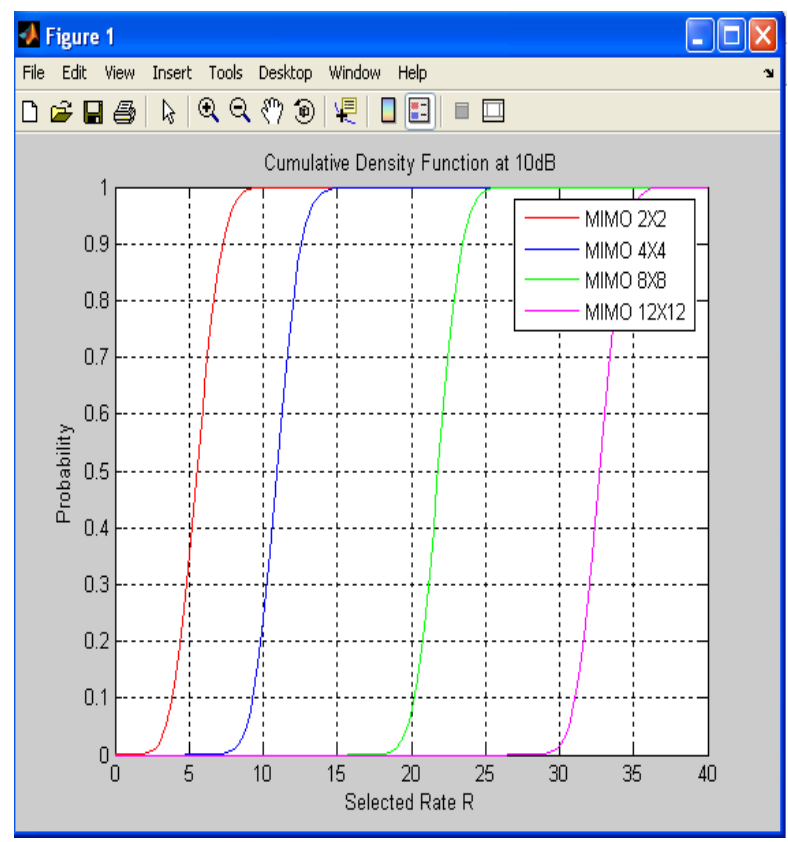

Fig 6: Comparison of MIMO Systems when SNR = $10 \mathrm{db}$
4.3 Case 3: Analysis of MIMO Systems for $\mathrm{SNR}=20 \mathrm{db}$

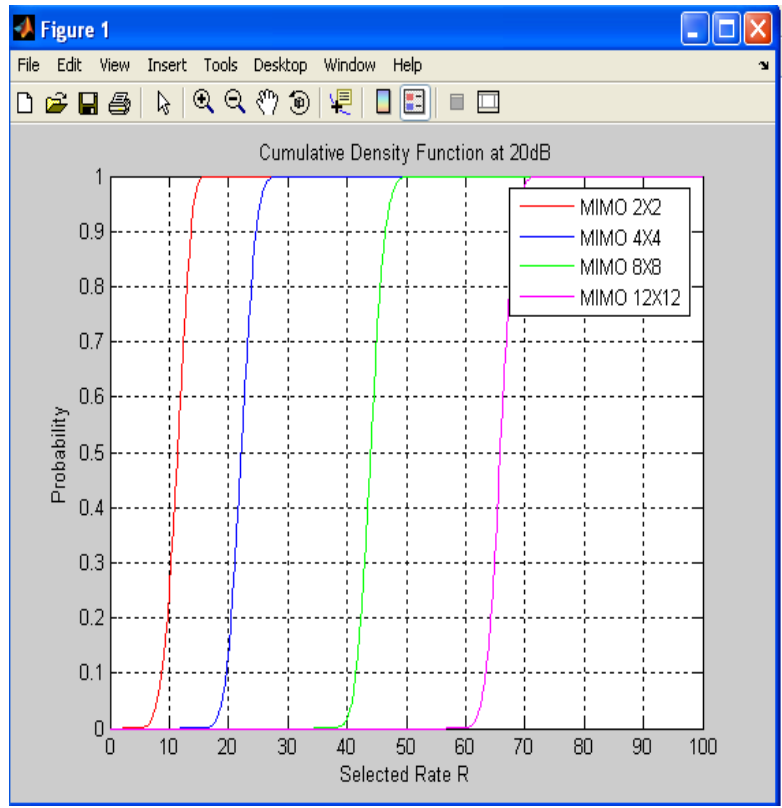

Fig 7: Comparison of MIMO Systems when SNR =20 db

\subsection{Case 4: Analysis of MIMO Systems for $\mathrm{SNR}=40 \mathrm{db}$}

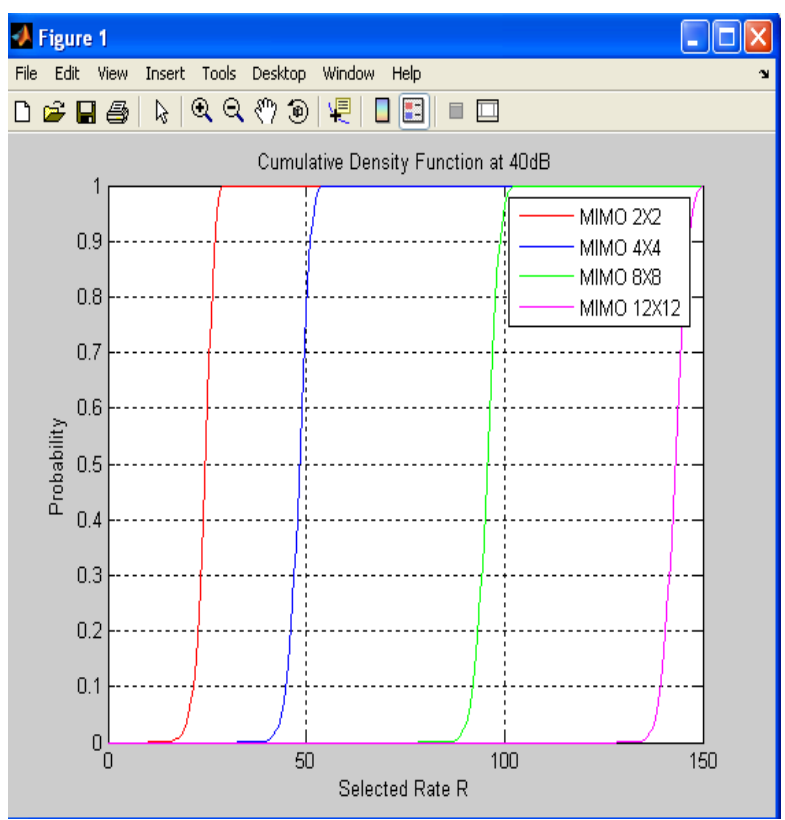

Fig 8: Comparison of MIMO Systems when SNR = $40 \mathrm{db}$ 


\subsection{Case 5: Analysis of MIMO Systems for $\mathrm{SNR}=60 \mathrm{db}$}

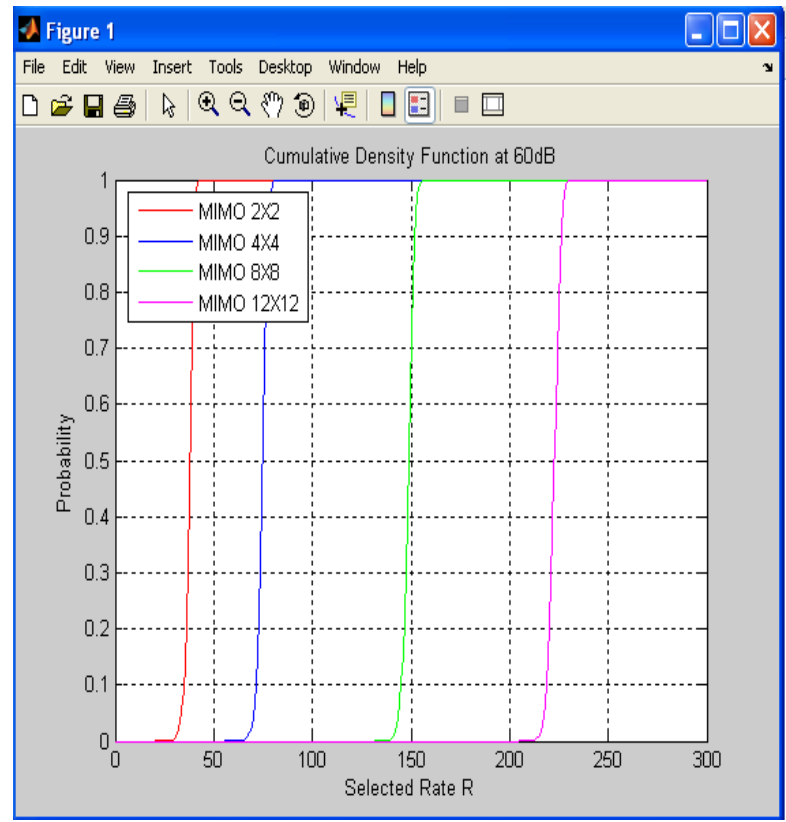

Fig 9: Comparison of MIMO Systems when SNR = $60 \mathrm{db}$

In this section, MIMO Systems have been analyzed by plotting a probability versus channel rate graph. The above results have been demonstrated by varying the values of SNR. It is well examined by the graphs that the channel capacity increases with increase in number of antennas as the channel capacity is maximum for $12 \times 12$ antenna and minimum for $2 \times 2$ antenna configuration for different values of SNR

\section{CONCLUSION}

In this paper, an introduction to a future technology called Multiple-Input-Multiple-Output (MIMO) has presented. This technology plays a vital role in wireless communication standard such as 4G, WiMax and IEEE 802.11n (WiFi) as it provides high data rates and low bit error rates. In the above section, the MIMO Systems are compared with different configurations by changing the values of SNR. After analyzing the above graphs it can be concluded that at low value of SNR such as $0 \mathrm{db}$ there is not much difference between the channel capacities of different MIMO Systems but at high value of SNR the difference between the channel capacities is highly noticeable.

\section{REFERENCES}

[1] Yang Wen Liang, "Ergodic and Outage Capacity of Narrowband MIMO Gaussian Channels", Department of Electrical and Computer Engineering, The University of British Columbia, Vancouver, British Columbia.

[2] David Gesbert, Mansoor Shafi, Da-shan Shiu, Peter J. Smith, and Ayman Naguib, 2003. "From Theory to Practice: An Overview of MIMO Space-Time Coded Wireless Systems", IEEE Journal on Selected Areas in Communications, Vol. 21, No. 3, April 2003.

[3] Luis G. Ord'onnez, Daniel P. Palomar, and Javier R. Fonollosa 2010. "On the Diversity, Multiplexing, and Array Gain Tradeoff in MIMO Channels", ISIT 2010, Austin, Texas, U.S.A., June 13 - 18, 2010.

[4] Shahab Sanayei and Aria Nosratinia, 2004. "Antenna Selection in MIMO Systems" University of Texas at Dallas, IEEE Communications Magazine.

[5] X Gu, X-H Peng and G C Zhang, 2006. "MIMO systems for broadband wireless Communications", BT Technology Journal, Vol 24 No 2, April 2006.

[6] Tim Brown, Elisabeth De Carvalho, Persefoni Kyristi, 2012. "Practical Guide to MIMO Radio Channel",Wiley

[7] Arif Khan, Rein Vesilo,"Tutorial on SISO and MIMO Channel Capacities"

[8] G. J. Foschini and M. J. Gans, "On limits of wireless communication in a fading environment when using multiple antennas," Wireless Personal Communications, vol. 6, pp. 311-335, Mar. 1998.

[9] Tolga M. Duman and Ali Ghrayeb "Coding for MIMO Communication System", Wiley

[10] D. W. Bliss, Keith W. Forsythe, and Amanda M. chan "MIMO Wireless Communication", Lincoln Labt. Journal, Vol. 15, No. 1, 2005

[11] Muhammad Razin Ibn Azad "Multiple Input Multiple Output", Thesis ,29thMay , 2012

[12] Prasad Rayi, Sarat Chandra ch, "Performance Evaluation of Channel Capacity In MIMO System", International Journal of Engineering Research and Applications (IJERA) ISSN 2248-9662, Vol. 1, Issue 4, pp.1871-1878.

[13] BengtHolter, "On The Capacity of the MIMO Channel-A Tutorial Introduction". 